\title{
A novel MLH1 mutation in a Japanese family with Lynch syndrome associated with small bowel cancer
}

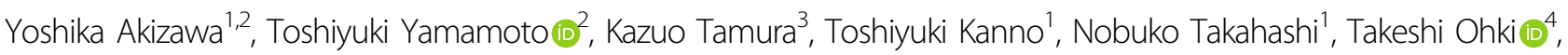 \\ Teppei Omori ${ }^{5}$, Katsutoshi Tokushige ${ }^{5}$, Masakazu Yamamoto ${ }^{4}$ and Kayoko Saito ${ }^{2}$
}

\begin{abstract}
Lynch syndrome is a genetic disorder related to cancer predisposition, including colorectal cancer, endometrial cancer, and ovarian cancer. Germline mutations in mismatch repair genes, including MLH1, MSH2, MSH6, and PMS2, are responsible for this condition. Cancer tissue specimens resected from small bowel adenocarcinoma in a Japanese patient showed decreased expression of MLH1 and PMS2 by immunohistochemistry testing. Finally, a novel MLH1 mutation, c.1833dup, was identified in this patient.
\end{abstract}

Colorectal cancers (CRCs) are involved in major types of cancer in Japan, and more than 49,000 Japanese people with CRCs died in $2015^{1}$. Approximately $20 \sim 25 \%$ of patients with CRCs show a family history, and genetic factors account for $5 \sim 15 \%$ of all CRC patients ${ }^{2}$. Some genetic cancer syndromes are involved in this condition. In 1966, Lynch et al. reported two large families with hereditary nonpolyposis colorectal cancer (HNPCC; MIM $\# 114500)$ to differentiate it from another inherited form of $\mathrm{CRC}^{3,4}$. However, patients with HNPCC often present other cancers, suggesting a predisposition to multiple cancers. Thus, Lynch syndrome (LS; MIM \#120435) is now a widely used alternative term.

LS is generally inherited as an autosomal dominant trait. Familial clustering of CRCs, an excess of synchronous or metachronous CRCs, and other extra colonic malignancies are often observed in the same family with LS. Germline mutations in mismatch repair (MMR) genes, including $M L H 1, M S H 2, M S H 6$, and PMS2, are related to LS, with a penetrance of approximately $80 \%$ for CRCs, $60 \%$ for

\footnotetext{
Correspondence: Yoshika Akizawa (akizawa.yoshika@twmu.ac.jp)

'Department of Obstetrics and Gynecology, Tokyo Women's Medical

University, Tokyo, Japan

${ }^{2}$ Institute of Medical Genetics, Tokyo Women's Medical University, Tokyo, Japan

Full list of author information is available at the end of the article.
}

endometrial cancer, and less than $20 \%$ for other cancers ${ }^{5}$. Generally, molecular diagnosis will be offered to patients who fulfill the clinical diagnostic criteria as defined by the Amsterdam I/II criteria or revised Bethesda guidelines ${ }^{4,6}$. If the patients receive a final diagnosis by molecular analysis, such information will be useful not only for themselves but also for their family members. Presymptomatic family members carrying the same variants are at risk of developing some cancers. Thus, they will be recommended for periodic health check-ups. However, if at-risk family members do not show the same variants, they will be released from the high-risk health check-ups.

Recently, we saw a new patient with LS who presented a novel mutation of $M L H 1$. The proband (II-2) is a 43-yearold Japanese male. He was referred to the department of gastroenterology in our hospital for a medical check-up owing to microcytic anemia and persistent fecal occult bleeding. Endoscopy examination was performed, and small bowel cancer was suspected. Finally, a diagnosis of adenocarcinoma was obtained by endoscopic small bowel biopsy. Laparoscopic partial intestinal resection was performed under the diagnosis of jejunal cancer. Pathological examination confirmed moderately differentiated adenocarcinoma, and postoperative chemotherapy using capecitabine was administered. Later, recurrent cancer was 


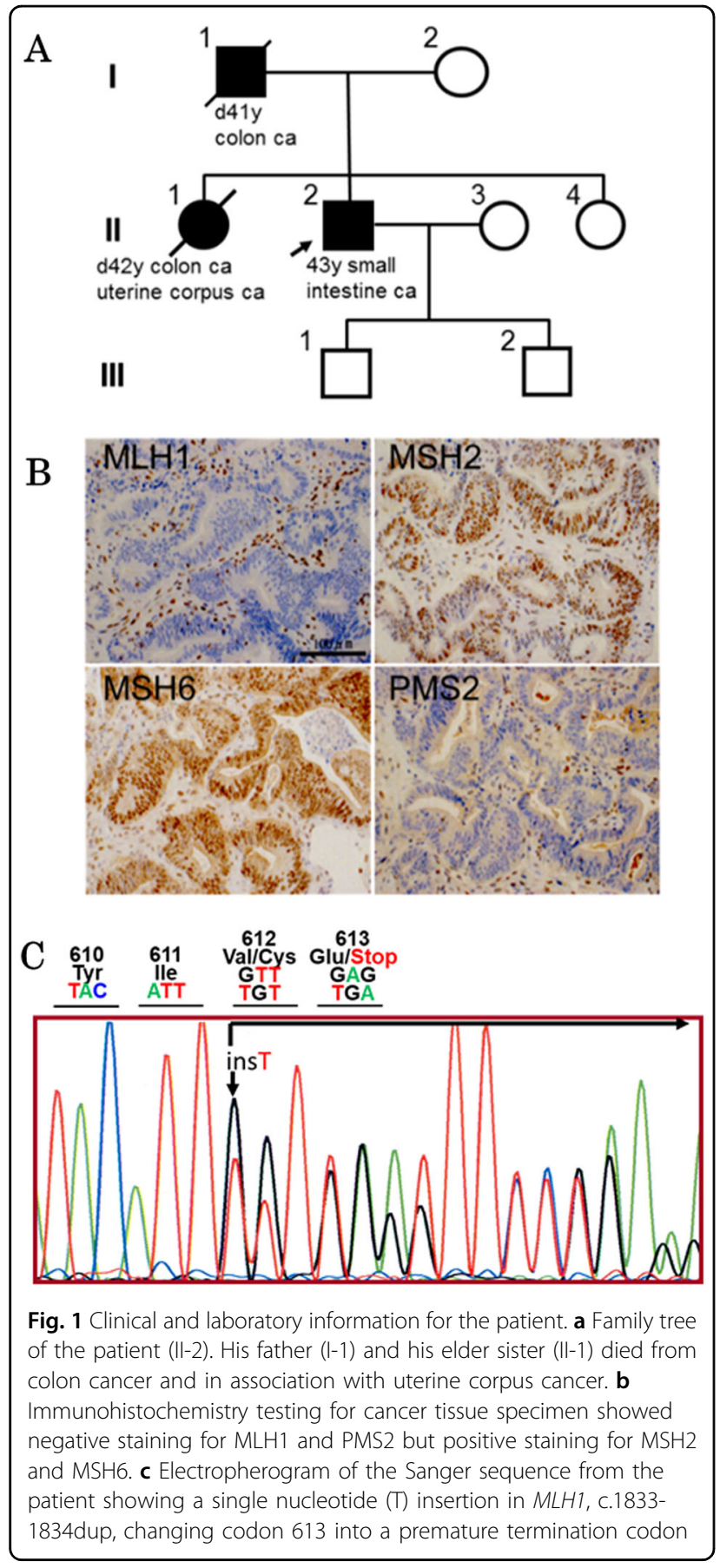

identified in the mesenteric lymph node. Again, the small bowel was partially resected after lymph node dissection.

This patient has a remarkable family history. His father (I-1) died of rectal cancer at 41 years of age, and his elder sister (II-1) died of cancer of the corpus uteri and rectal cancer at 42 years of age (Fig. 1a). According to Amsterdam criteria II, we suspected LS as a potential candidate diagnosis. Three family members were affected, and all of them were first-degree relatives; they were diagnosed before 50 years of age, and two successive generations were affected. To confirm the clinical diagnosis, a molecular examination was performed.

This study was performed in accordance with the Declaration of Helsinki and was approved by the ethical committee of the Tokyo Women's Medical University. After obtaining written informed consent, blood samples and tissue specimens from extracted small bowel cancer tissue were collected. First, immunohistochemistry testing was performed, and loss of MLH1 and PMS2 expression was determined (Fig. 1b). This suggested the involvement of the $M L H 1$ gene because MLH1 protein forms a dimer with PMS2 protein ${ }^{7,8}$. For confirmation, Sanger sequencing analysis of $M L H 1$ was performed according to previously reported methods ${ }^{9}$. For this analysis, genomic DNA was extracted using a QIAamp DNA Mini Kit (Qiagen, Hilden, Germany). Finally, a possible pathogenic mutation, NM_000249.3 (MLH1_v001):c.1833dup [p. Val612Cysfs*2] (Fig. 1c), which leads to a premature termination codon, was identified (Fig. 1c). This mutation has not been registered in the InSiGHT variant database (https://www.insight-group.org/variants/databases/), in which 1344 MLH1 variants are currently registered. ClinVar (https://www.ncbi.nlm.nih.gov/clinvar/) and the professional human gene mutation databases (HGMD) (http://www.hgmd.cf.ac.uk/ac/index.php) were also checked, and this variant was not included. Therefore, c.1833dup was considered a novel mutation.

Among four MMR genes, three genes (MLH1, MSH2, and MSH6) shared 96\%, and MLH1 mutations were the most prevalent ${ }^{10}$. The sensitivity of immunohistochemistry testing to detect MMR gene mutations has been reported to be $45 \%^{11}$. Therefore, identification of the MLH1 mutation in this patient showing loss-of-function for MLH1 and PMS2 by immunohistochemistry testing is reasonable.

According to the database, most of the reported pathogenic variants in MMR genes lead to nonsense or premature termination ${ }^{12}$, which suggests that the pathogenic mechanism of MMR genes is mainly derived from a loss-offunction. Loss-of-heterozygosity likely occurs in cancer cells by second hit mutations in somatic cells. By this mechanism, the expression of MMR genes would be decreased, as shown by immunohistochemistry testing for cancer tissues.

In the present patient, the first disease manifestation was small bowel cancer, which is not rare in patients with $\mathrm{LS}^{13}$, ${ }^{14}$. After careful genetic counseling and obtaining permission from the patient, his genetic information was disclosed to his partner and younger sister (II-3 and II-4).The younger sister (II-4) declined to be genotyped herself and was referred for periodic medical check-ups, which is acceptable because she has the right not to know her genotype $^{15,16}$. More time and more supports may be necessary for her for consideration ${ }^{17,18}$. Regarding the offspring of the 
present patient under the age of adulthood, genetic counseling about their genetic testing to confirm their carrier status should be provided in the future.

In this study, we successfully identified a novel $M L H 1$ mutation in a Japanese patient with LS. This genetic information will be useful if the patient's offspring are willing to be genotyped in the future.

\section{HGV Database}

The relevant data from this Data Report are hosted at the Human Genome Variation Database at https://doi.org/10.6084/m9.figshare.hgv.2324.

\section{Acknowledgements}

We are grateful to the patients and their families for their cooperation. We thank Dr. Naohiro Tomita for his participation in the immunohistochemistry and Dr. Hiroshi Tanaka and Akemi Yamauchi for their English support during the proofreading of our manuscript.

\section{Author details}

${ }^{1}$ Department of Obstetrics and Gynecology, Tokyo Women's Medical University, Tokyo, Japan. ${ }^{2}$ Institute of Medical Genetics, Tokyo Women's Medical University, Tokyo, Japan. ${ }^{3}$ Magor in Science, Graduate School of Science and Engineering Research, Kinki University, Higashiosaka, Japan. ${ }^{4}$ Department of Surgery, Institute of Gastroenterology, Tokyo Women's Medical University, Tokyo, Japan. ${ }^{5}$ Institute of Gastroenterology, Department of Internal Medicine, Tokyo Women's Medical University, Tokyo, Japan

\section{Conflict of interest}

The authors declare that they have no conflict of interest.

\section{Publisher's note}

Springer Nature remains neutral with regard to jurisdictional claims in published maps and institutional affiliations.

Received: 11 April 2018 Revised: 15 May 2018 Accepted: 16 May 2018. Published online: 21 June 2018

\section{References}

1. JSCCR. JSCCR Guidelines 2016 for the Treatment of Colorectal Cancer. (Kanehara Shuppan, Tokyo, 2016).

2. de la Chapelle, A. Genetic predisposition to colorectal cancer. Nat. Rev. Cancer 4, 769-780 (2004)
3. Lynch, H. T., Shaw, M. W., Magnuson, C. W., Larsen, A. L. \& Krush, A. J. Hereditary factors in cancer. Study of two large midwestern kindreds. Arch. Intern. Med. 117, 206-212 (1966).

4. Tiwari, A. K., Roy, H. K. \& Lynch, H. T. Lynch syndrome in the 21st century: clinical perspectives. QM 109, 151-158 (2016).

5. Lynch, H. T. \& de la Chapelle, A. Hereditary colorectal cancer. N. Engl. J. Med. 348, 919-932 (2003).

6. Lindor, N. M. et al. Recommendations for the care of individuals with an inherited predisposition to Lynch syndrome: a systematic review. JAMA 296, 1507-1517 (2006).

7. Bellizzi, A. M. \& Frankel, W. L. Colorectal cancer due to deficiency in DNA mismatch repair function: a review. Adv. Anat. Pathol. 16, 405-417 (2009).

8. Markow, M., Chen, W. \& Frankel, W. L. Immunohistochemical pitfalls: common mistakes in the evaluation of Lynch syndrome. Surg. Pathol. Clin. 10, 977-1007 (2017).

9. Yamaguchi, T. et al. Comparison of clinical features between suspected familial colorectal cancer type $X$ and Lynch syndrome in Japanese patients with colorectal cancer: a cross-sectional study conducted by the Japanese Society for Cancer of the Colon and Rectum. Jpn. J. Clin. Oncol. 45, 153-159 (2015).

10. Peltomaki, P. \& Vasen, H. Mutations associated with HNPCC predisposition-Update of ICG-HNPCC/NSiGHT mutation database. Dis. Markers 20, 269-276 (2004)

11. Steinhagen, E. et al. Systematic immunohistochemistry screening for Lynch syndrome in early age-of-onset colorectal cancer patients undergoing surgical resection. J. Am. Coll. Surg. 214, 61-67 (2012).

12. Sjursen, W., McPhillips, M., Scott, R. J. \& Talseth-Palmer, B. A. Lynch syndrome mutation spectrum in New South Wales, Australia, including 55 novel mutations. Mol. Genet. Genom. Med. 4, 223-231 (2016).

13. Haanstra, J. F. et al. Prevalence of small-bowel neoplasia in Lynch syndrome assessed by video capsule endoscopy. Gut 64, 1578-1583 (2015).

14. Schulmann, K. et al. HNPCC-associated small bowel cancer: clinical and molecular characteristics. Gastroenterology 128, 590-599 (2005).

15. Takala, T. The right to genetic ignorance confirmed. Bioethics 13, 288-293 (1999).

16. Berkman, B. E. \& Hull, S. C. The "right not to know" in the genomic era: time to break from tradition? Am. J. Bioeth. 14, 28-31 (2014).

17. White, V. B. et al. Genetic testing for hereditary breast cancer: the decision to decline. Am. Surg. 84, 154-160 (2018).

18. Kanga-Parabia, A., Gaff, C., Flander, L., Jenkins, M. \& Keogh, L. A. Discussions about predictive genetic testing for Lynch syndrome: the role of health professionals and families in decisions to decline. Fam Cancer (2018). Feb 20. https://doi.org/10.1007/s10689-018-0078-2 [Epub ahead of print]. 